\title{
ENHANCED VOF-BASED DIRECT NUMERICAL SIMULATIONS OF SLUG FLOW BOILING WITHIN MICRO-CHANNELS WITH SMOOTH AND FINNED HEATED WALLS
}

\author{
Emanuele Teodori ${ }^{*}$, Manolia Andredaki ${ }^{2}$, Anastasios Georgoulas ${ }^{2}$, Ana Moita ${ }^{1}$, Antonio Moreira ${ }^{1}$, \\ Marco Marengo ${ }^{2}$ \\ ${ }^{1}$ IN+ Center for Innovation, Technology and Policy Research, Instituto Superior Técnico, Universidade de \\ Lisboa, Av. Rovisco Pais, Lisbon 1049-001, Portugal \\ ${ }^{2}$ Advanced Engineering Centre, School of Computing, Engineering and Mathematics, Cockcroft Building, \\ Lewes Road, University of Brighton, Brighton BN2 4GJ, UK
}

\begin{abstract}
Flow boiling in micro-channels constitutes an effective cooling method which has been proven experimentally to be able to remove significantly high heat fluxes, while keeping the component of interest at an appropriate operating temperature. Plenty of experimental works exist in literature so far, but appropriate models to predict flow boiling heat transfer are still lacking or they are based on unrealistic assumptions. In the present investigation, direct numerical simulations of vapour slug growth associated with flow boiling within micro-channels are performed, utilising an enhanced Volume of Fluid (VOF) based method that accounts for spurious currents reduction and phase-change. The effect of various micro-structured finned patterns in the heated wall of the channels is numerically investigated. The main aim is to give further insight on the effect of surface structuring on flow boiling in the slug flow regime. The utilised, enhanced VOF-based model is implemented in OpenFOAM CFD toolbox. Initially, comparison with literature available numerical results for a straight wall circular microchannel is carried out. After the proposed comparison, different geometrical configurations of the heated part of the utilised channel are implemented, keeping the same operating and initial conditions. In more detail, various fin patterns of rectangular cross-section are introduced in the heated wall of the channel. The analysis of the results reveals in order to have an increase in the heat transfer coefficient an optimum geometric configuration is needed.
\end{abstract}

KEY WORDS: Two phase flow, heat transfer enhancement, numerical simulation, OpenFOAM, micro-channels, finned walls

\section{INTRODUCTION}

Flow boiling within micro-channels can constitute an attractive cooling solution for high density power components, due to its ability of removing high heat fluxes up to $50 \mathrm{MW} / \mathrm{m}^{2}$, when for example is applied to cooling of Insulated-Gate Bipolar Transistors (IGBT) or other components as reviewed in [1].

However, the physics involved in flow boiling within micro-channels is still a matter of experimental investigations which are frequently in disagreement in terms of identification and correlation of fundamental parameters, as pointed out in several reviews of the topic such as [2], [3], [4] .Due to this, mechanistic modelling of flow boiling in micro-channels is still a matter of discussion despite several models proposed in literature e.g [5].

Modelling and physical description of flow boiling is even more complicated when for example structured micro-channels are used aiming to increase the heat transfer coefficient. In this context fins or pin fins were 
found effective in several studies e.g. [6], [7], [8], [9]. The combined effect of enlarged heat transfer area, increased number of nucleation sites and other beneficial effects from the bubble dynamics point of view, were identified as some of the reasons for an increased heat transfer coefficient of these kind of surfaces, compared to smooth channels.

Besides the aforementioned processes, other underpinned heat transfer mechanisms and alteration of the fluid dynamics were observed by some authors and correlated with an enhancement of heat transfer coefficient. Ma et al. [6], claimed that forced convection plays a vital role in the case of pin-finned channels. In addition, the growth and movement of the bubbles in the confined gaps between subsequent fins, can generate microconvection mechanisms and form thin liquid layers for evaporation, making the profile of fins become an effective heat transfer area. Differently, the authors in [7] noticed wakes induced downstream the channel due to the formation of bubbles on the sidewalls of the pin fins. These wakes disrupted the boundary layer in the region adjacent to the bubble, locally increasing the heat transfer coefficient. In [8], the authors stated that adding micro-cone pin fins on the micro-channel wall surface, generated numerous stable nucleation sites by the formation of lots of tiny re-entrant cavities. Moreover, they increased the capillary force introducing wicking effects for the liquid rewetting, delaying the local dry-out and maintaining high heat transfer coefficients, even at high heat fluxes and vapor qualities. Bigham et al. [9], focused on liquid film evaporation in the slug flow regime, highlighting that micro structuring the surface, increases the film/solid contact area and the passage time of the liquid slug consequently increasing heat removal rates.

The disagreement found in literature in terms of underlying micro-scale phenomena in structured microchannels can be correlated with limitations in the measurement techniques. To tackle this issue, in the recent years, the use of CFD codes has been extended to the analysis of three-dimensional, multi-phase flows. Nowadays the numerical simulation of boiling heat transfer has become possible, for a wide range of applications as well as spatial and temporal scales. This, thanks to the growing computing capabilities, amount of available computing resources and the rapid development of modern numerical methods for the simulation of multiphase flows. One of the main difficulty in reproducing two phase flows, is the proper reproduction of the liquid-vapour or liquid-gas interface topology. In this context, the most widely used interface "capturing" methods in the literature for the numerical reproduction of flow boiling within micro-channels, are the Level Set (LS) method (e.g. [10], [11], [12], [13]) and the Volume of Fluid (VOF) method (e.g. [14] [15] [16] [17] [18]). Utilising such "Direct Numerical Simulation" (DNS) methods for the capturing the interface between the liquid and vapour phases, interesting micro-scale heat transfer phenomena can be identified and quantified. For example, Yan and $\mathrm{Zu}$ [14], revealed that a relative cross-flow of liquid past bubbles takes place generating vortices near the interface which may enhance the local heat transfer rates. [16], highlighted that leading and sequential bubbles, in slug flow boiling, interact thermally and hydrodynamically due to the evaporation process, thus possessing different growth rates, velocities and thicknesses of the thin liquid films trapped between the bubbles and the channel wall. Besides thin film evaporation, the authors pointed out the presence of a recirculating vortex just after the tail of a leading bubble, within the liquid slug trapped between two subsequent bubbles, that significantly enhances the sensible heat transfer between the wall and the bulk liquid.

However, all the aforementioned studies, focused only on smooth channels. According to the Authors' best knowledge, only the work of Lee et al. [13], studied the effect of fins in the heated walls of micro-channels, numerically. Focusing on elongated bubbles flow, the authors emphasized that an optimization of the fins distribution is required to increase the heat transfer coefficient. They showed that a particular distribution of the fins could lead to an augmented thin liquid film/solid contact area and hence to higher heat flux removal rates due to thin film evaporation.

It is clear from all these investigations, that there is a lack of numerical data referring to structured micro channels. The present investigation presents simulations of slug flow boiling on smooth and finned circular micro-channels, conducting a parametric analysis of the main geometrical characteristics in the case of the finned wall channels. An enhanced VOF-based model, developed in the general context of OpenFOAM, is utilised for the computations. The proposed model has been presented, validated and applied in a previous investigation [19]. 


\section{METHODS}

\subsection{Governing equations}

We hereby present the governing equations of the problem, for more detailed information concerning the evaporation model one can refer to [19].

The mass conservation is given as:

$$
\nabla \cdot(\rho \vec{U})=\dot{\rho}
$$

Where $\mathrm{U}$ is the fluid velocity and $\rho$ is the bulk density. The source term on the right hand side accounts for phase change.

The momentum conservation follows the here reported formulation:

$$
\frac{\partial}{\partial t}(\rho \vec{U})+\nabla \cdot(\rho \vec{U} \vec{U})-\nabla \cdot\left\{\mu\left[\nabla \vec{U}+(\nabla \vec{U})^{T}\right]\right\}=-\nabla \mathrm{p}+\vec{f}_{S T}+\vec{f}_{g}
$$

where $\mathrm{p}$ is the pressure and $\mu$ is the bulk dynamic viscosity. Volumetric gravitational forces are represented as $\vec{f}_{g}$. while $\vec{f}_{S T}$ represents the volumetric surface tension forces

The Continuum Surface Force (CFS) method proposed in [20] is used to model the surface tension as a volumetric force,

$$
\vec{f}_{S T}=\sigma \kappa(\nabla \alpha)
$$

where $\sigma$ is the surface tension and $\kappa$ the curvature of the interface.

Reproduction of the curvature required a smoothing procedure of the interface developed in [21], to account for an adequate level of spurious currents suppression. The procedure involves the calculation of the interface curvature using smoothed volume fraction values $\tilde{\alpha}$ that are obtained from the initially calculated volume fraction field $\alpha$, smoothing it over a finite region near the interface.

$$
\kappa=\nabla \cdot\left(\frac{\nabla \widetilde{\alpha}}{|\nabla \tilde{a}|}\right)
$$

The proposed smoothing is achieved by the application of a Laplacian filter which can be described by the following equation:

$$
\widetilde{\alpha}_{p}=\frac{\sum \alpha_{\mathrm{f}} \mathrm{S}_{\mathrm{f}}}{\sum \mathrm{S}_{\mathrm{f}}}
$$

Where the subscripts $P$ and $f$ denote the cell and the face index respectively while $\alpha \_f$ is the linear interpolated value of the volume fraction at the face center and $S_{\mathrm{f}}$ is the surface of the face. More details on the smoothing method and its validation can be found in [21]. The smoothed volume fraction is used only for the calculation of the curvature all the other equations are based on the initially calculated (non-smoothed) values of the volume fraction.

Energy balance is conserved according to following equation:

$$
\frac{\partial}{\partial t}\left(\rho c_{p} T\right)+\nabla \cdot\left(\vec{U} \rho c_{p} T\right)-\nabla \cdot(\lambda \nabla T)=\dot{h}
$$


where $c_{p}$ is the bulk heat capacity, $T$ the temperature field and $\lambda$ the bulk thermal conductivity. The source term $\dot{h}$ considers in part the contribution of the enthalpy of evaporation.

For the advection of the volume fraction $\alpha$ the following formulation stands:

$$
\frac{\partial \alpha}{\partial t}+\nabla \cdot(\alpha \vec{U})-\nabla \cdot\left(\alpha(1-\alpha) U_{r}\right)=\frac{\dot{\rho}}{\rho} \alpha
$$

Interphase sharpening is important in two phase flow simulation of immiscible fluids. The artificially introduced compression term in eq. $4 \nabla \cdot\left(\alpha(1-\alpha) U_{r}\right)$ is used in Open FOAM to achieve interface sharpening. $U_{r}$ is the artificial compression velocity, more details are given in [19].

The bulk fluid properties $\gamma$ are computed as the averages over the liquid $\gamma_{l}$ and vapour phases $\gamma_{v}$ weighted with the volume fraction $\alpha$ :

$$
\gamma=\alpha \gamma_{l}+(1-\alpha) \gamma_{v}
$$

Phase change is modelled according to the work of [22]. Based on this, the calculation of evaporating mass flux at the liquid/vapour interface is based on the following equation:

$$
\mathrm{j}_{\text {evap }}=\frac{\mathrm{T}_{\mathrm{int}}-\mathrm{T}_{\mathrm{sat}}}{\mathrm{R}_{\mathrm{int}} \mathrm{h}_{\mathrm{lv}}}
$$

where $T_{\text {sat }}$ is the saturation temperature, $T_{\text {int }}$ is the temperature at the interface, resulting from the evaporation model, $\mathrm{h}_{\mathrm{lv}}$ is the latent heat of evaporation at the saturation temperature. and $\mathrm{R}_{\mathrm{int}}$ is the interfacial heat resistance calculated by the following equation based on the assumptions of [23]

$$
R_{\text {int }}=\frac{2-f}{f} \frac{\sqrt{2 \pi R_{\text {gas }}}}{h_{l v}^{2}} \frac{T_{\text {sat }}^{3 / 2}}{\rho_{v}},
$$

Where $f$ is an evaporation/condensation coefficient which is a fitting parameter with a value ranging between 0 and 1 . Following the approach of [19], a value of 1 was used in the presented simulations.

\subsection{Simulation parameters}

Simulations are performed with the finite-volume-based CFD solver OpenFOAM (v.2.2.1). PISO (PressureImplicit with Splitting of Operators) scheme is applied for pressure velocity coupling. Transient terms are discretised using a second order, bounded, implicit scheme (Euler). A maximum Courant number of 0.2 is set. The calculation time step is controlled by the courant number and varies from approximatively $10^{-9}$ to $10^{-6} \mathrm{sec}$. The gradient terms are discretized using a second order, Gaussian integration with linear interpolation (Gauss linear). For the divergence terms different discretisation schemes are applied for each term in the equations. For convection term of Eq. (2) a "Gauss-upwind" scheme, in Eq (7) for the term $\nabla \cdot(\alpha \vec{U})$ "Gaus-vanLeer" scheme, while for $\nabla \cdot\left(\alpha(1-\alpha) U_{r}\right)$ a "Gauss interfaceCompression" scheme is used. All Laplacian terms are discretised using the "Gauss Linear Corrected" scheme while the divergence term in Eq.(6) is discretised using a "Gauss linear" scheme. This combination of discretization schemes was found as optimal to maintain a balance between accuracy, convergence and numerical stability during the computation.

\subsection{Simulation set-up}

Slug flow in a horizontal circular microchannel is simulated under saturation conditions for a straight channel and for three different finned channels. Only the fluid region is simulated in this work as the conjugate heat transfer problem is not solved. Given this only the geometrical properties of the fins are of interest. The width of the fins $\mathrm{W}_{\text {fin }}$ and the distance between fins centers $S_{\text {fin }}$ was kept constant and equal to $\mathrm{W}_{\text {fin }}=0.555 \mathrm{~mm}$ and 
$S_{\text {fin }-1}=1.11 \mathrm{~mm}$ the height of the fins $\mathrm{H}_{\text {fin }}$ was varied as follows: $\mathrm{H}_{\text {fin }}=12.5 \mu \mathrm{m}, \mathrm{H}_{\text {fin }}=6.25 \mu \mathrm{m}$ and $\mathrm{H}_{\text {fin }}=18.75$ $\mu \mathrm{m}$.

For all the configuration studied the following description stands. An axis-symmetric domain corresponding to a wedge geometry of $5^{\circ}$ and of diameter $\mathrm{D}=500 \mu \mathrm{m}$ is used. The channel develops its extension along the $\mathrm{x}$ axis and comprises of three sections: an initial adiabatic section of $4 \mathrm{~mm}$ in length followed by a heated section of $11 \mathrm{~mm}$ (straight or finned) and a final adiabatic section of $8 \mathrm{~mm}$. The fluid chosen for the simulations is the refrigerant $\mathrm{R} 245 \mathrm{fa}\left(\mathrm{T}_{\mathrm{sat}}=304 \mathrm{~K}\right.$ at $\left.\mathrm{p}_{\mathrm{sat}}=1 \mathrm{~atm}\right)$. At the channel inlet saturated liquid flows with an imposed velocity along the $x$ axis of $0.413 \mathrm{~m} / \mathrm{s}$. At the heated section a constant heat flux of $5000 \mathrm{~W} / \mathrm{m}^{2}$ is imposed and a no-slip boundary condition is prescribed all along the channel walls. At the outlet a constant boundary condition is imposed for the pressure while a zero gradient condition is imposed for temperature and velocity. After solving the velocity and temperature fields at the beginning of the simulation for a liquid only steady state case, vapour slugs are patched at distance $0.5 \mathrm{~mm}$ from the entrance of the channel at time instants defined by a prescribed frequency of bubble generation of $\mathrm{f}=104 \mathrm{~Hz}$. The slugs have a length of $1.5 \mathrm{~mm}$, height of $0.46 \mathrm{~mm}$ corresponding to a volume of $0.206 \mathrm{~mm}^{3}$. Fig 1, depicts the initial condition for the simulations in a straight channel together with the boundary conditions

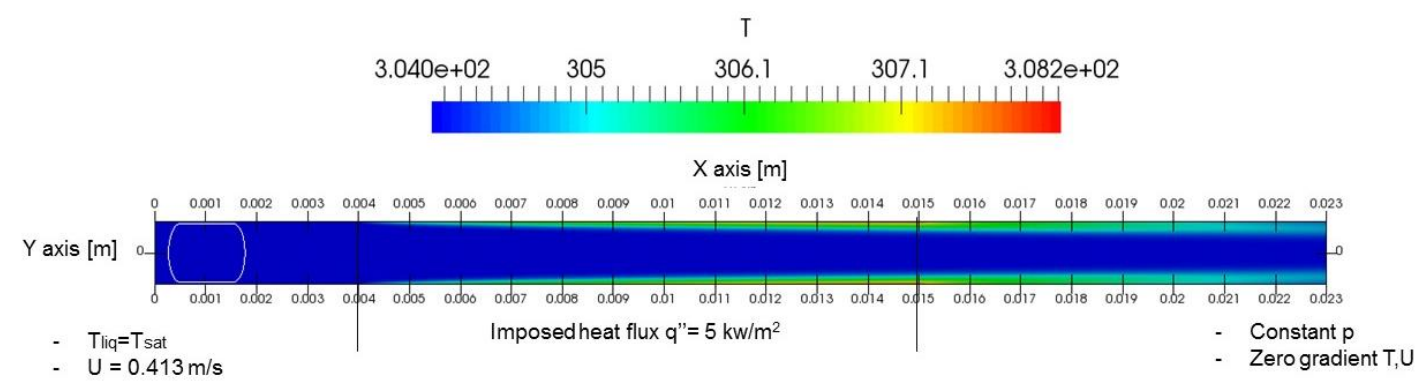

Fig. 1 Schematic of the initial flow conditions and boundary conditions used for the simulations

\subsection{Slug flow in straight channel}

The proposed numerical model was already benchmarked in [19], [21] for two phase flows in adiabatic cases and pool boiling. However, the results obtained for slug flow in a straight channel are qualitatively compared with the numerical work of Magnini and Thome [17] , from which the initial flow conditions were taken. To do so six bubble cycles are simulated assuring periodical conditions. Two different mesh configurations were used. the first comprises uniform hexahedral cells of dimensions $1.67 \mu \mathrm{m}^{2}$ and a total of around $2 \times 10^{6}$ cells, the second, coarser, comprises again uniform hexahedral cells of dimensions $8 \mu \mathrm{m}^{2}$ and a total of around $2 \times 10^{5}$ cells. The cells dimension of the coarser mesh was considered adequate to proper capture the liquid film between the vapor slug and the wall.

In figure $2(a, b, c)$ the scaled temperature and velocity field at the beginning of the sixth bubble cycle is illustrated for the work of Magnini and Thome [17] (figure 2a), for the finer mesh (figure 2b) and for the coarse mesh (figure 2c). From a first analysis one can conclude that good agreement was found in terms of temperature and velocity fields. Also, the bubble length was only slightly over predicted in the case study here reported being $13 \%$ higher the one reported Magnini and Thome [17] when the fine mesh is used and 50\% higher when the coarse mesh is used. However, for the coarse mesh, the average heat transfer coefficient $h_{a v}=q^{\prime \prime} /\left(\overline{T_{w}}-T_{s a t}\right)$ measured probing the average wall temperature over time $\overline{T_{w}}$ at $9.755 \mathrm{~mm}$ from the channel inlet was $2285.7 \mathrm{~W} / \mathrm{m}^{2} \mathrm{~K}$ which is $18 \%$ lower than the one reported in the numerical simulation of Magnini and Thome [17], but closer to the experimental data the proposed authors used to compare. Therefore, the coarse mesh is adopted since it is considered accurate enough, for the purposes of the present numerical investigation. 


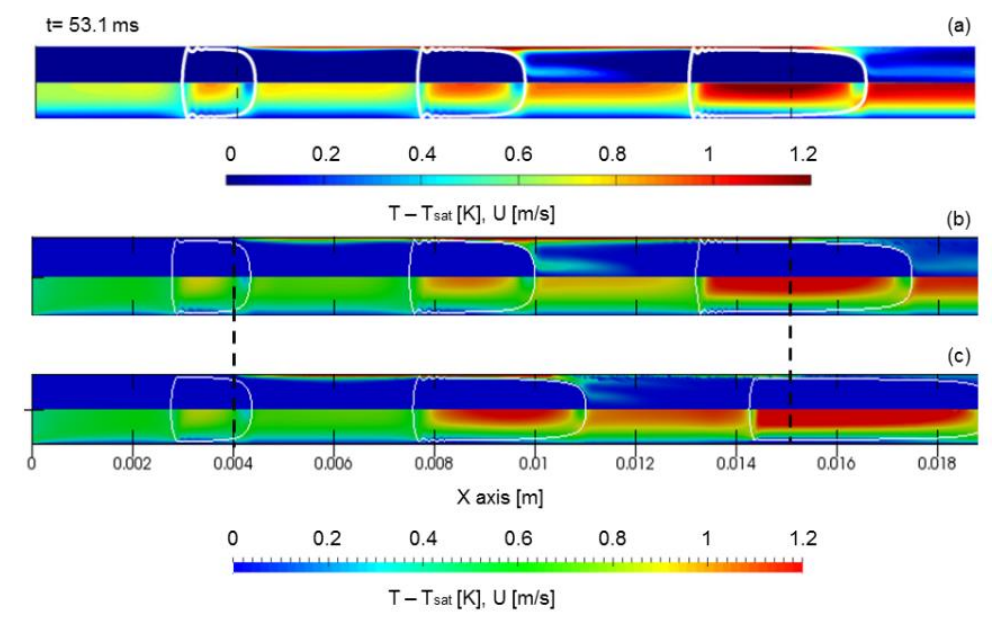

Fig. 2 Scaled velocity and temperature fields at time $53.1 \mathrm{~ms}$. (a) From the work of Magnini and Thome [17] (b) present work with fine mesh (c) present work with coarse mesh. The dashed lines indicate the heated section

\subsection{Effect of fins height on Slug flow boiling in finned channels}

The effect of the fins height on the global heat transfer coefficient was studied in the present work. The global heat transfer coefficient was obtained for the fifth bubble cycle according to the following procedure: three time instances during all the period in which the bubble crosses the channel were chosen. The first corresponding to the instant in which the bubble nose reaches the beginning of the heated section the second taken as half the time the bubble takes to cross the heated section and the third corresponding to the moment in which the bubble tail leaves the heated section. For each time step the temperature is calculated as average of the temperature profile along the heated wall surface. Finally, the three temperatures obtained are also averaged giving the final wall temperature $\overline{T_{w g l}}$ for the calculation of a global heat transfer coefficient according to the formulation:

$$
h_{g l}=\frac{q^{\prime \prime}}{\overline{T_{w g l}}-T_{s a t}}
$$

In Figure 3, the global heat transfer coefficient for finned micro channels divided by the one of the straight channel is reported versus the fin height. It is possible to observe that among the finned channels studied only the one with $\mathrm{H}_{\mathrm{fin}}=18.75 \mu \mathrm{m}$ could express higher heat transfer coefficients if compared to the smooth channel. From the results reported one can conclude that geometric optimization is usually required when using finned channels similarly to what inferred in Lee et al. [13]. In the cases of the present investigation the height of the fins is relatively small if compared to the typical dimensions of the vapour slug. Hence the fins interact with the slug in a different way from what is reported in Lee et al. [13]. To highlight this Figure 4 depicts a comparison of the vapour slug shapes taken at $10.7 \mathrm{~ms}$ after the entrance of the heated region.

From a qualitative point of view it can be inferred that channels with $\mathrm{H}_{\text {fin }}=6.25 \mu \mathrm{m}$ and $\mathrm{H}_{\text {fin }}=12.5 \mu \mathrm{m}$ are affecting the slug shape in such a way that a slight increase of the film thickness can be noticed if compared to the smooth channel. Hence vaporization could be inhibited and in fact smaller vapour slugs of length equal to $3.2 \mathrm{~mm}$ were observed for these geometries. On the other side for the $\mathrm{H}_{\text {fin }}=18.75 \mu \mathrm{m}$ channel the slug penetrates the space in between the fins resulting in a diminished thickness of the liquid film. Consistently both the straight and the $\mathrm{H}_{\text {fin }}=$ $18.75 \mu \mathrm{m}$ channels are characterized by vapour slugs of length of $3.6 \mathrm{~mm}$. Consequently, vaporization is not the dominant mechanism of heat transfer since despite the improved heat transfer coefficient of surface $\mathrm{H}_{\text {fin }}=18.75$ $\mu \mathrm{m}$ the length of the bubble, proportional to the evaporation rate, was found equal to the one of the straight channel. To understand the best performance of the channel $\mathrm{H}_{\mathrm{fin}}=18.75 \mu \mathrm{m}$ one can observe the slug nose position which is at $11.75 \mathrm{~mm}$ for the straight channel at around $11.55-11.6 \mathrm{~mm}$ for the $\mathrm{H}_{\text {fin }}=6.25 \mu \mathrm{m}$ and $\mathrm{H}_{\text {fin }}=12.5 \mu \mathrm{m}$ channels 
and at $12.1 \mathrm{~mm}$ for the $\mathrm{H}_{\text {fin }}=18.75 \mu \mathrm{m}$ channel. The presented results emphasize that the finned channel $\mathrm{H}_{\text {fin }}=$ $18.75 \mu \mathrm{m}$ is characterized by the higher speed of the vapour slug as it crosses through the channel. Concluding one could infer that the higher velocity of the slug could be correlated then with dynamic phenomena occurring within the liquid that could lead to the higher values of heat transfer coefficient for the channel $\mathrm{H}_{\text {fin }}=18.75 \mu \mathrm{m}$. Nevertheless, a deeper analysis of the flow within the channel should be performed to properly infer on underlying mechanisms of heat transfer promoting heat removal in particular geometries of fins.

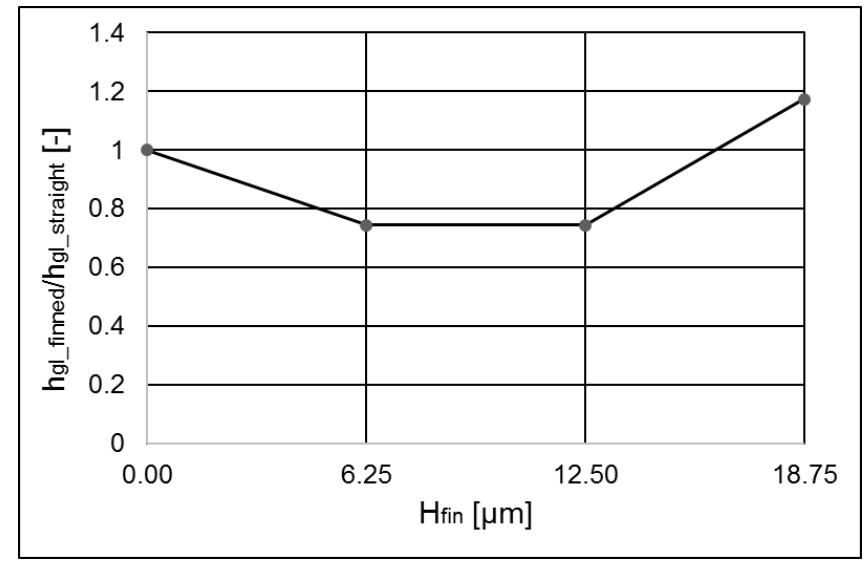

Fig. 3 Ratio between global heat transfer coefficient of finned micro channels and straight channel versus fins height

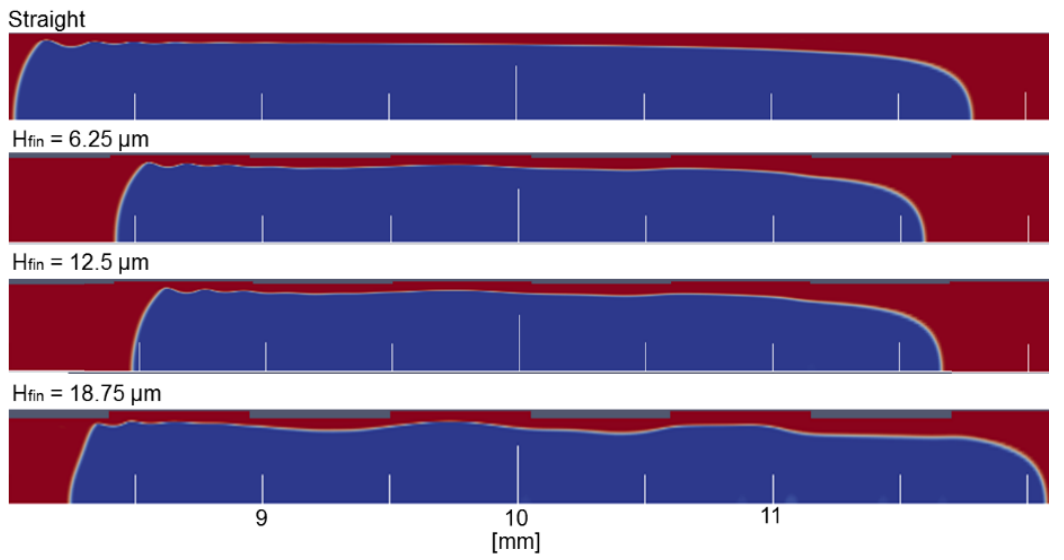

Fig. 4 Vapour slug shape at $t=10.7 \mathrm{~ms}$ after the entrance of the vapour slug in the heated section of the channel for the different channel geometries studied

\section{CONCLUSIONS}

In the present work CFD simulations of slug flow boiling in straight and finned micro channel were presented. The effect of the fins height on the heat transfer coefficient was addressed revealing that optimization was required in order to positively affect the heat removal process. Beside vaporization other mechanisms of heat transfer are playing a vital role during flow boiling in structured micro channels. Deeper investigation is required to shed light on these mechanisms of heat transfer.

\section{ACKNOWLEDGMENT}

The Authors would like to acknowledge UK's Engineering and Physical Science Research Council support through the grant EP/P013112/1 as well as the ESA MAP Project INWIP. The authors would also like to 
acknowledge the contribution of the High-Performance Computing facility of the School of Computing Engineering and Mathematics, in the University of Brighton. The authors are grateful to Fundação para a Ciência e Tecnologia (FCT) for partially financing the research under the framework of the project UID/EEA/50009/2013 which also supported P. Pontes and E. Teodori with a research fellowship. A. S. Moita acknowledges FCT for financing her contract through the IF 2015 recruitment program (IF 00810-2015).

\section{REFERENCES}

[1] T. Karayiannis e M. Mahmoud, "Flow boiling in microchannels: Fundamentals and applications," Applied Thermal Engineering, vol. 115, pp. 1372-1397, 2017.

[2] S. Garimella e C. Sobhan, "TRANSPORT IN MICROCHANNELS -A CRITICAL REVIEW," Annual Review Heat Transfer, vol. 13, pp. 1-50, 2003.

[3] S. Bertsch, E. Groll e S. Garimella, "Review and Comparative Analysis of Studies on Saturated Flow Boiling in Small Channels," Nanoscale and Microscale Thermophysical Engineering, vol. 12, n 3, pp. 187-227, 2008.

[4] J. Thome, "Boiling in microchannels: a review of experiment and theory," International Journal of Heat and Fluid Flow, vol. 25, pp. 128-139, 2004.

[5] J. Thome, V. Dupont e A. Jabobi, "Heat Transfer Model for Evaporation in Microchannels. Part I: Presentation of the Model," International Journal of Heat and Mass Transfer , vol. 47, pp. 3375-3385, 2004.

[6] A. Ma, J. Wei, M. Yuan e J. Fang, "Enhanced flow boiling heat transfer of FC-72 on micro-pin-finned surfaces," International Journal of Heat and Mass Transfer, vol. 52, p. 2925-2931, 2009.

[7] S. Krishnamurthy e Y. Peles, "Flow Boiling Heat Transfer on Micro Pin Fins Entrenched in a Microchannel," Journal of Heat Transfer, vol. 132, pp. 1-10, 2010.

[8] D. Deng, W. Wana, Y. Qin, J. Zhang e X. Chu, "Flow boiling enhancement of structured microchannels with micro pin fins," International Journal of Heat and Mass Transfer, vol. 105, p. 338-349, 2017.

[9] S. Bigham, A. Fazeli e S. Moghaddam, "Physics of microstructures enhancement of thin film evaporation heat transfer in microchannels flow boiling," Scientific Reports, vol. 7, n 4475, p. 11, 2017.

[10] A. Mukherjee e S. Kandlikar, "Numerical simulation of growth of a vapor bubble during flow boiling of water in a microchannel," Microfluid Nanofluid, p. 137-145, 2005.

[11] W. Lee e G. Son, "Bubble Dynamics and Heat Transfer During Nucleate Boiling in a Microchannel," Numerical Heat Transfer, Part A:Applications: An International Journal of Computation and Methodology, vol. 53, n 10, pp. 1074-1090, 2008.

[12] A. Mukherjee, S. Kandlikar e Z. Edel, "Numerical study of bubble growth and wall heat transfer during flow boiling in a microchannel," International Journal of Heat and Mass Transfer, vol. 54, pp. 3702-3718, 2011.

[13] W. Lee, G. Son e H. Yoon, "Direct numerical simulation of flow boiling in a finned microchannel,” International Communications in Heat and Mass Transfer, vol. 39, pp. 1460-1466, 2012.

[14] Y. Yan e Y. Zu, "Numerical Simulation of Bubbles Deformation, Flow, and Coalescence in a Microchannel Under PseudoNucleation Conditions," Heat Transfer Engineering, vol. 32, pp. 1182-1190, 2011.

[15] M. Magnini, B. Pulvirenti e J. Thome, "Numerical investigation of hydrodynamics and heat transfer of elongated bubbles during flow boiling in a microchannel," International Journal of Heat and Mass Transfer, vol. 59, pp. 461-471, 2013.

[16] M. Magnini, B. Pulvirenti e J. Thome, "Numerical investigation of the influence of leading and sequential bubbles on slug flow boiling within a microchannel," International Journal of Thermal Sciences, vol. 71, pp. 36-52, 2013.

[17] M. Magnini e J. Thome, "Computational Study of Saturated Flow Boiling Within a Microchannel in the Slug Flow Regime," Journal of Heat Transfer, vol. 138, pp. 1-12, 2016.

[18] Z. Pan, J. Weibel e S. Garimella, "A saturated-interface-volume phase change model for simulating flow boiling," International Journal of Heat and Mass Transfer, vol. 93, pp. 945-956, 2016.

[19] A. Georgoulas, M. Andredaki e M. Marengo, "An Enhanced VOF Method Coupled with Heat Transfer and Phase Change to Characterise Bubble Detachment in Saturated Pool Boiling," Energies, vol. 10, pp. 1-35, 2017.

[20] J. Brackbill, D. Kothe e C. Zemach, “A continuum method for modeling surface tension,” Journal of Computational Physics, vol. 100, p. 335-354, 1992.

[21] A. Georgoulas, P. Koukouvinis, M. Gavaises e M. Marengo, "Numerical investigation of quasi-static bubble growth and detachment from submerged orifices in isothermal liquid pools: The effect of varying fluid properties and gravity levels," International Journal of Multiphase Flow, vol. 74, pp. 59-78, 2015.

[22] S. Hardt e F. Wondra, "Evaporation Model for Interfacial Flows Based on a Continuum-Field Representation of the Source Terms," Journal of Computational Physics, vol. 227, n 11, pp. 5871-5895, 2008.

[23] R. Schrage, A Theoretical Study of Interphase Mass Transfer, New York: Columbia University Press, 1953. 